\title{
Pregnancy with Preexisting Total Uterine Prolapse
}

\section{Adeviye Elçi Atılgan ${ }^{1 *}$ and Şükriye Leyla Altuntaş ${ }^{2}$}

${ }^{1}$ Department of Urogynaecology, Faculty of Medicine, Istanbul Medipol University Hospital, Istanbul, Turkey ${ }^{2}$ Department of Obstetry and Gynecology, Faculty of Medicine, Istanbul Medipol University Hospital, Istanbul, Turkey

*Corresponding author: Adeviye Elçi Atılgan, MD, Asistant Professor, Department of Urogynaecology, Faculty of Medicine, istanbul Medipol Üniversity, Istanbul, Bağcılar, Turkey, Tel: +905057553783

\begin{abstract}
Management of pregnancy with uterine prolapse is challenging. Physician may encounter scenario which endanger both mother and baby during pregnancy, at labor and also after birth. Here, we present conservative management of a pregnancy with preexisting total uterine prolapse up to term. We performed cesarean section due to previous delivery by cesarean section. Interestingly, prolapse did not recur after delivery at two years follow up period. Further reports are needed to conclude as pre-existent uterine prolapse may resolve spontaneously at pregnancy and also may not recur after cesarean section.
\end{abstract}

\section{Keywords}

Clinical obstetrics, Uterine prolapse, Pregnancy, Obstetrics, Management, Urogynecology

\section{Introduction}

Pelvic organ prolapse (POP) may first manifest during pregnancy or may pre-exist. Each condition is risky for mother and fetus. The main risks for pregnant women with POP are preterm labor, urinary retention, urinary infection, sepsis, cervical laceration, uterine rupture and even maternal death [1,2]. For the fetus, the risks are serious like neurological complications, bone fracture due to dystocia at labor, pulmonary insufficiency, multiorgan failure due to preterm delivery, septicemia and maybe fetal death [3]. Fortunately, POP occurrence both primarily during pregnancy and pre-existent are rare [4].

In developed countries, the incidence of POP with pregnancy has decreased significantly in relation to the decrease in parity [5]. High parity $(\geq 5)$ is one of the strongest risk factor for POP development [6]. Also, severity of symptoms increase with high numbers of vaginal deliveries and high weight of infant delivered vaginally [7]. There is a few datain the literature that prolapse persists or recurs after delivery $[8,9]$. Herein we present a woman with pre-existing stage four uterine prolapse according to Pelvic Organ Prolapse Quantification System (POPQ) [10]. She had had an appoinment for Laparascopic Sacrohysteropexy procedure for prolapse. However, she got pregnant unintentionally up to appoinment and prolapse resolved spontaneously during pregnancy. Interestingly, prolapse didn't recur after delivery by cesarean section at 2 years follow-up period.

\section{Case Presentation}

A 39-years-old woman presented at our urogynecology unit complaining of vaginal bulging and protruding a mass out of her vagina. After urogynecologic examination she was diagnosed with four-degree uterine prolapse according to standard Pelvic Organ Prolapse Quantification (POP-Q) system (Figure 1). Examination was performed both lithotomy and standing position with an empty bladder and an empty rectum to detect stage ofpelvic organ prolapse (POP) exactly as recommended [11]. Also a Sim's speculum was used to retract the anterior and posterior vaginal walls to assess all vaginal compartment for prolapse. The six defined points ( $A a, B a, C, D, A p, B p)$ that are considered while recording the POP-Q, which are used to report the extent of descent or prolapse of the anterior vaginal wall, vaginal apex, posterior wall and three measurements; 


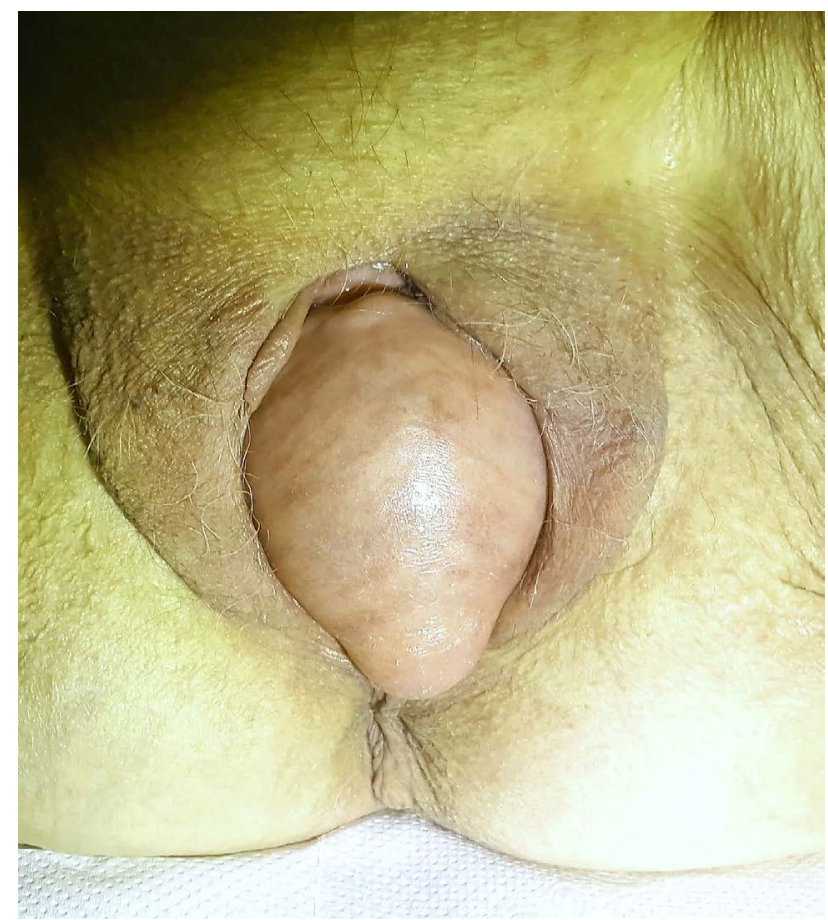

Figure 1: Patient with uterine prolapsed.

Table 1: A sample of fullfilled $3 \times 3$ grid used for $P O P-Q$ clinical documentation at 12 weeks of gestation [11].

\begin{tabular}{|l|l|l|}
\hline Aa:+2 & Ba:+3 & C:+7 \\
\hline Gh:4 & Pb:4 & Tvl:10 \\
\hline Ap:+3 & Bp:+3 & D:+7 \\
\hline
\end{tabular}

\section{POPQ: Measurements}

The locations of the six defined points when the prolapse is fully reduced.

Anterior vaginal wall:

1. Point Aa: A point located in the midline of the anterior vaginal wall three (3) $\mathrm{cm}$ proximal to the external urethral meatus. The potential range of position of Point Aa relative to the hymen is -3 , indicating no anterior vaginal $P O P$, to $+3 \mathrm{~cm}$ which is full prolapse.

2. Point Ba: A point that represents the most distal (ie, most dependent) position of any part of the upper anterior vaginal wall (between the vaginal cuff or anterior vaginal fornix and Point $\mathrm{Aa}$ ). Point $\mathrm{Ba}$ coincides with Point $\mathrm{Aa}(-3 \mathrm{~cm})$ in a woman who has no anterior POP. In a woman with severe POP, Ba coincides with Point $\mathrm{C}$.

\section{Upper vagina:}

3. Point C: A point on either the most distal (ie, most dependent) edge of the cervix or the leading edge of the vaginal cuff (hysterectomy scar).

4. Point D: The posterior fornix in a woman who still has a cervix.

\section{Posterior vaginal wall:}

5. Point Ap: A point located in the midline of the posterior vaginal wall three (3) $\mathrm{cm}$ proximal to the hymen. The potential range of position of Point $A p$ relative to the hymen is -3 to $+3 \mathrm{~cm}$.

6. Point Bp: A point that represents the most distal position of any part of the upper posterior vaginal wall (between the vaginal cuff or posterior vaginal fornix and Point Ap).
Three further descriptive landmarks and measurements:

1. The genital hiatus $(\mathrm{GH})$ is measured from the middle of the external urethral meatus to the posterior margin of the hymen.

2. The total vaginal length (TVL) is the length of the vagina $(\mathrm{cm})$ from posterior fornix to hymen when Point $C$ or $D$ is reduced to its full normal position.

3. The perineal body $(P B)$ is measured from the posterior margin of the hymen to the mid-anal opening.

Genital hiatus (Gh); Perineal body $(\mathrm{Pb})$ and total vaginal length (TVL) relate to a $3 \times 3$ grid used for clinical documentation [11] (Table 1).

The points are measured during maximal Valsalva or cough in relation to the hymen. When the point descends to the hymen it is measured as $0 \mathrm{~cm}$, if it remains above the hymen it is measured in centimeters and described as negative integers and if it descends beyond the hymen it is measured in centimeters and described as positive integers [11]. This grid was used in the entire follow-up process of the patient.

In her previous history, she had one vaginal delivery with 3300 grams at termwithout episiotomy, forcep or vacuum 4 years ago and one cesarean delivery due to macrosomic fetus (4800 grams) 2 years ago. She stated that she was taken to planned ceserean section at $39^{\text {th }}$ weeks before the labor not started and postoperative process were all normal. She had added that had breastfeeding her baby for 8 months. Her complaints of vaginal bulging started approximately one years ago and increased day by day. Her Body Mass Index (BMI) was 24 $\mathrm{kg} / \mathrm{m}^{2}$ and she was from East Asian ethnicity. She was an academician not require heavy-lifting work which may increase the risk of POP.

Other systems had no problems on physical examination with transabdominal ultrasound assessment. She stated desire for surgical correction of the genital prolapse with sterilization. We offered her Laparoscopic Sacrohysteropexy procedure concomitant with Laparoscopic Bilateral Tubal Ligation. However, she became pregnant before the scheduled appointment on the day of operation.

The first prenatal visit was performed at $6^{\text {th }}$ weeks of gestational age and a single alive fetus was observed with transvaginal ultrasounography. We re-examined her vaginally and according to POPQ system fourth degree uterine prolapse was observed similar like before pregnancy. At $12^{\text {th }}$ weeks visit, we recommended inserting a pessary, but she refused due to the discomfort of a foreign body in her vagina and potential risk of infection. Then, we recommended bed rest in a moderate Trendelenburg position and use of moisturizer to prevent cervical dryness. Close follow-up with vaginal examination and translabial ultrasound at each visit, prolapse persisted up to 26 weeks of gestational age.

When 32 weeks control, antenatal corticosteroids 
were administered to accelerate fetal lung maturation due to the potential risk of preterm birth. Cesarean section was performed with spontaneous rupture of membranes at 37 weeks. The operation was uneventful and concomitant bilateral tubal ligation was performed with Pomeroy's technique. We didn't carry out uterosacral ligament plication at the time of cesarian section to correct the uterine prolapse. The postoperative period was uneventhful and vaginal examination was performed especially for potential risk of prolapse recürrence. However, prolapse was not observed and two days later she was discharged with her healthy baby.

One week later and 6 weeks later postoperative controls were performed with transabdominal ultrousound assessment and vaginal examinations. Uterine prolapse was still not observed.

Patient continued to be followed up every 6 months with vaginal examination and transvaginal ultrasound performed to detect any residue bulging or mild recurrent prolapse at our urogynecology unit. Overall, at the end of 2 years follow-up period, uterine prolapse did not recur.

\section{Discussion}

Physiological increases in cortisol and progesterone levels during pregnancy lead to softening of pelvic tissues as well as apical cardinal and uterosacral supportive ligaments. For this reason, acute onset of POP in pregnancy is a more common condition than pregnancy with pre-existing POP $[3,8]$. Routine surgery for POP correction during pregnancy is not recommended [12]. There are potential risks for both mother and fetus from the operation. In addition, the long-term consequences are unknown [12]. There are a few case reports about Laparascopic Sacrohysterteropexy or Promontohysteropexy performed at first trimestr of pregnancy [12-15].

Use of a pessary is recommended until labor $[1,4,5]$. On the other hand, pessary may cause infection, cervical ulceration or may exacerbate cervical edema. Cervical infection may lead to spontaneous abortion or preterm delivery. There was a maternal death reported due to infected pessarylead to sepsisin the literature [2]. In this case, the patient refused to insert a pessary. We followed-up her with conservative approaches like bed rest, slight Trendelenburg position, and avoiding heavy lifting. We observed prolapse was resolved during pregnancy occasionally.

When it is time for labor, the decision about delivery method is controversial. Inability to maintain appropriate cervical dilatation due to prolapse may result in cervical dystocia. During the ongoing process of labor, obstructive labor, as well as cervical laceration and even rupture of the lower uterine segment may ocur. The decision must be individualized by considering possible complications. Our case underwent cesarean section with the indication of previous delivery by cesarean section.
There is a case of a 33-year-old woman with twin gestation who admitted to clinic with labor contractions and total uterine prolapse at 33 weeks of gestation. An emergent cesarean section was performed for the indication of acute fetal distress. At the same operation, following cesarean delivery, abdominal hysteropexy using rectus fascia strips was performed successfully by Karatayli, et al. [16].

The different point that pay attention is, at 33 weeks of gestation total uterine prolapse was present here whereas in our case prolapsus was resolved. So the authors had to perform abdominal hysteropexy using rectus fascia strips at $\mathrm{C}$-section session. A smilar case was reported by Meydanlı, et al. [17]. A 30-years-old multipara woman with stage 3 uterine prolapse was admitted to the hospital with uterine contractions at 35 weeks of gestation. They performed cesarean hysterectomy and sacrocolpopexy procedures at same session. They suggested as cesarean hysterectomy option especially for women who have completed their families and are suffering from severe pelvic organ prolapse [17].

Most Obstetricians prefer cesarean delivery for this reason. However, there are reported cases about successful births via the vaginal route [15]. Prolapse usually persists or recurs after delivery even with cesarean section $[4,15]$. However, our case did not recur during 2 years follow up. There is also one more case reported with prolapse which didn't recur after cesarean section in the literatüre [18]. We can't say cesarean delivery protects women from prolapse. The exact mechanism isn't known, but adhesions between uterosacral, cardinal or broad ligaments and pelvic structures during the ceserean healing process may be possible factor for prevent recurrence. Also herein bilateral tubal ligation was carried out on woman requesting sterility, not for uterine prolapse correction. We do not suspect that uterine prolapse did not recur after delivery as a result of the tubal ligation. We think that prolapse spontaneously resolved mostly due to pregnancy itself. Pregnancy may be a chance for preexisting uterine prolapse cases by regenerating the uterine ligaments. However there is limited study on this subject in the literatüre.

\section{Conclusion}

A pregnant woman with pelvic organ prolapse can be managed conservatively up to delivery. For these cases, strict prenatal follow-up and lifestyle suggestions are inevitable. Although we observed prolapse did not recur after cesarean delivery, we need many more randomized controlled studies to conclude as pregnancy may be a chance for prexisting uterine prolapse cases and prolapse may not recur after cesarean delivery.

\section{Conflict of Interest}

Author state having no conflict of interest and received any financial support. 


\section{Patient's Consent}

Patient's permission was obtained to publication of this case report.

\section{Funding}

None.

\section{References}

1. Yousaf S, Haq B, Rana T (2011) Extensive uterovaginal prolapse during labor. J Obstet Gynaecol Res 37: 264-266.

2. Hill PS (1984) Uterine prolapse complicating pregnancy. A case report. J Reprod Med 29: 631-633.

3. Skurupii DA, Sonnyk EG, Sizonenko VM (2018) Multiorgan failure syndrome in newborns: Role of social and anatomico-functional features (literature review). Wiad Lek 71: 777780 .

4. Tsikouras P, Dafopoulos A, Vrachnis N, Iliodromiti Z, Bouchlariotou S, et al. (2014) Uterine prolapse in pregnancy: Risk factors, complications and management. J Matern Fetal Neonatal Med 27: 297-302.

5. Rusavy Z, Bombieri L, Freeman RM (2015) Procidentia in pregnancy: Asystematic review and recommendations for practice. Int Urogynecol J 26: 1103-1109.

6. Kurt S, Canda MT, Bal M, Tasyurt A (2018) Are there any preventable risk factors for women who had surgery for Pelvic Organ Prolapse and stress Urinary Incontinence? Pak J Med Sci 34: 874-878.

7. Swift S, Woodman P, O'Boyle A, Kahn M, Valley M, et al. (2005) Pelvic Organ Support Study (POSST): the distribution, clinical definition, and epidemiologic condition of pelvic organ support defects. Am J Obstet Gynecol 192: 795-806.

8. Mohamed-Suphan N, Ng RK (2012) Uterine prolapse complicating pregnancy and labor: A case report and literature review. Int Urogynecol J 23: 647-650.
9. Yogev Y, Horowitz ER, Ben-Haroush A, Kaplan B (2003) Uterine cervical elongation and prolapse during pregnancy: An old unsolved problem. Clin Exp Obstet Gynecol 30: 183-185.

10. Auwad W, Freeman FR, Swift FS (2004) Is the pelvic organ prolapse quantification system (POPQ) being used? A survey of members of the International Continence Society (ICS) and the American Urogynecologic Society (AUGS). Int Urogynecol J 15: 324-327.

11. Madhu C, Swift S, Moloney-Geany S, Drake MJ (2018) How to use the Pelvic Organ Prolapse Quantification (POP-Q) system? Neurourology and Urodynamics 37: S39-S43.

12. Matsumoto T, Nishi M, Yokota M, Ito M (1999) Laparoscopic treatment of uterine prolapse during pregnancy. Obstet Gynecol 93: 849.

13. Haliloglu Peker B, Ilter E, Peker H, Celik A, Gursoy A, et al. (2018) Laparoscopic Sacrohysteropexy in a Woman at 12 Weeks' Gestation. J Minim Invasive Gynecol 25: 11461147.

14. Pirtea L, Balint O, Secosan C, Ilina R, Grigoras D (2017) Laparoscopic Promontohysteropexy During Pregnancy. J Minim Invasive Gynecol 24: 1073-1074.

15. Eddib A, Allaf MB, Lele A (2010) Pregnancy in a woman with uterine procidentia: A case report. J Reprod Med 55: 67-70.

16. Karataylı R, Gezginç K, Kantarcı AH, Acar A (2013) Successful treatment of uterine prolapse by abdominal hysteropexy performed during cesarean section. Arch Gynecol Obstet 287: 319-322.

17. Meydanli MM, Ustün Y, Yalcin OT (2006) Pelvic organ prolapse complicating third trimester pregnancy. A case report. Gynecol Obstet Invest 61: 133-134.

18. Toy $\mathrm{H}$, Camuzcuoğlu $\mathrm{H}$, Aydın $\mathrm{H}$ (2009) Uterine prolapse in a 19 year old pregnant woman: A case report. J Turk Ger Gynecol Assoc 10: 184-185. 